\title{
Erratum to: Combining Pharmacological and Psychological Treatments for Binge Eating Disorder: Current Status, Limitations, and Future Directions
}

\author{
Carlos M. Grilo ${ }^{1}$ Deborah L. Reas ${ }^{2,3}$ • James E. Mitchell ${ }^{4,5}$
}

Published online: 23 July 2016

(C) Springer Science+Business Media New York 2016

Erratum to: Curr Psychiatry Rep (2016) 18: 55

DOI 10.1007/s11920-016-0696-z

On Page 9 of 11 of the published article, in the last paragraph the authors wrote:

"The limitations noted earlier for LDX (including contraindication for obesity and weight loss) potentially limit its relevance as a viable combination or additive strategy to enhance psychological treatments for BED."

The authors would like the above sentence to read:

"The limitations noted earlier for LDX (including the "Limitation of Use" that it is not indicated for weight loss and the "Warning" regarding the potential for abuse) potentially limit its relevance as a viable combination or additive strategy to enhance psychological treatments for BED."

The online version of the original article can be found at http://dx.doi. org/10.1007/s11920-016-0696-z.

Carlos M. Grilo

carlos.grilo@yale.edu

1 Department of Psychiatry, School of Medicine, Yale University, New Haven, CT, USA

2 Regional Department for Eating Disorders, Division of Mental Health and Addiction, Oslo University Hospital, Oslo, Norway

3 Department of Psychology, Faculty of Social Sciences, University of Oslo, Oslo, Norway

4 Neuropsychiatric Research Institute, Fargo, ND, USA

5 Department of Clinical Neuroscience, School of Medicine and Health Sciences, University of North Dakota, Fargo, ND, USA 